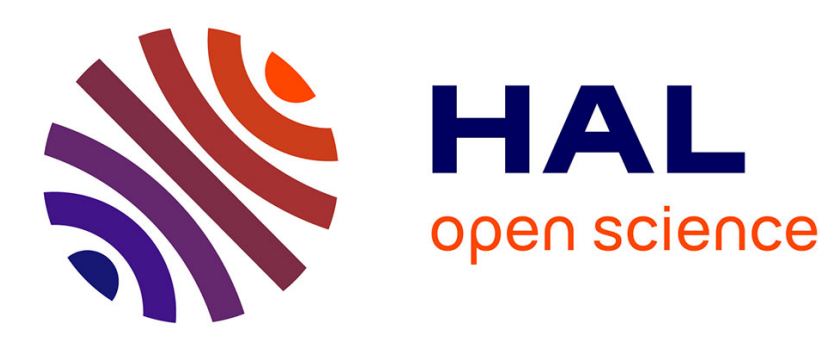

\title{
Chord length distributions of non convex bodies: Dumbbell and diabolo like-particles
}

Frédéric Gruy

\section{To cite this version:}

Frédéric Gruy. Chord length distributions of non convex bodies: Dumbbell and diabolo likeparticles. Computational Materials Science, 2015, 110, pp.1-9. 10.1016/j.commatsci.2015.07.052 . hal-03337029

\section{HAL Id: hal-03337029 \\ https://hal.science/hal-03337029}

Submitted on 7 Sep 2021

HAL is a multi-disciplinary open access archive for the deposit and dissemination of scientific research documents, whether they are published or not. The documents may come from teaching and research institutions in France or abroad, or from public or private research centers.
L'archive ouverte pluridisciplinaire HAL, est destinée au dépôt et à la diffusion de documents scientifiques de niveau recherche, publiés ou non, émanant des établissements d'enseignement et de recherche français ou étrangers, des laboratoires publics ou privés. 


\title{
Chord length distributions of non convex bodies:
}

\author{
dumbbell and diabolo like-particles
}

\section{Frédéric Gruy}

Ecole Nationale Supérieure des Mines, 158 Cours Fauriel, 42023, Saint-Etienne, France gruy@emse.fr

Phone: 0033477420202

Corresponding author

\begin{abstract}
The scattering of an electromagnetic wave by a particle is directly related to its Chord Length Distribution (CLD) in certain cases. Whereas the CLD of convex bodies, e.g. sphere, ellipsoids, cylinders..., can be easily calculated, few studies have been conducted on the CLD of non convex bodies which are more difficult to ascertain. Two non convex bodies built from two spheres, i.e. dumbbells and diabolos, were considered. Firstly, we describe original algorithms designed for calculating the intersection between a straight line and these particles. Then the corresponding CLDs are calculated by using the Monte-Carlo method. Analysis of the deviation of these CLDs from the CLD of the sphere can identify precisely the main features due to some local non convexity. The corresponding items are expressed as a function of a non convexity parameter.
\end{abstract}

Keywords: Chord Length Distribution (CLD), non convex bodies, light scattering, material characterization, Monte-Carlo simulation 


\section{Introduction}

Many manufacturers use solid micro-particles in suspension for various applications: ceramics, paintings, pharmaceutics, cosmetics, food and chemicals. Particle size can be evaluated by physical methods based on the scattering of an incident electromagnetic wave as it strikes the particle. The scattered wave depends on the particle morphology as well as on the ratio between the refractive indices of both the material and suspension medium. Depending on the material, the particle morphology and the selected method, the signal measured may be straightforwardly related to the Chord Length Distribution (CLD) of the set of the randomly orientated particles. This is applied for Small-Angle Scattering (SAS) measurements [1, 2], Focused Beam Reflectance Measurements (FBRM) [3], Spectral Turbidimetry, i.e extinction measurement [4].

Whereas a straight line passes just once through a convex body, it may intersect more than one time across a non convex body. As a consequence, there are two chord length distributions which can be defined as:

- The Multiple Chord Distribution (MCD) where each segment interval on the same line is considered as one chord length separately. FBRM measurements and experiments in the field of SAS are associated with MCD.

- The one chord distribution (OCD) where the sum of chord lengths for all intersected intervals is used as the definition of the chord length. Turbidity measurements are associated with OCD.

The CLD of convex and non-convex bodies has been studied mathematically [5-7]. Explicit expressions have been obtained for bounded 2D or 3D convex domains: disc, triangle, 
rectangle, regular polygon [8], sphere, hemisphere [9], cylinders of various cross sections [1011], spheroids [12], polyhedron [13-14].

However, to the best of our knowledge little attention has been paid to non convex bodies compared to convex ones. Mazzolo et al. [15] discussed the CLD in the context of reactor physics. They showed that some relations between lower moments of CLD and simple geometric properties as volume, surface, ... of the body remain valid for non-convex bodies whereas higher CLD moments do not obey the simple relations valid for convex bodies. Gille [16] studied the CLD of an infinitely long circular hollow cylinder that is a special case of non convex body; the corresponding calculation was based on basic mathematics. Vlasov [17] introduced the notion of signed chord distribution for convex and non-convex bodies. He started from the work of Dirac to reduce the six-dimensional integral of pairwise interaction potential for a convex body into a simpler expression including the CLD; then he extended this to a non convex body, showing that the expression of the integral is much more complicated than the one for a convex body. In the case of non convex body the integral can be decomposed into several terms (integrals), each related to the various segments of the given chord inside the non convex body. Vlasov formally deduced the expression of the CLD for the non-convex case. Gruy et al. analytically calculated the CLD of a two-sphere cluster [18] and a dumbbell [19].

There are some results linking CLD's with scattering experiments, particularly SASmeasurements, for non convex particles. This aspect has been analysed by Gille [20]. The geometric and physical quantity directly related to the SAS-intensity is the correlation function $\gamma(r)$. The function $r^{2} \gamma(r)$ is proportional to the distance distribution function. For instance, using cylindrical models, Gille [21] has studied the relation between non-convex particles and SAS. He derived explicitly the correlation function for two touching circular cylinders and deduced the second derivative $\gamma^{\prime \prime}$ that is proportional to the CLD for convex 
particles. When analysed he found five contributions for $\gamma^{\prime \prime}$ : each corresponding to a chord crossing a given sub-space: either traversing a sole cylinder, both cylinders or the space between the two cylinders... Therefore, this example proves that the CLD is related to the scattering intensity in a more complex manner for the case of non convex particles as opposed to convex particles. Kaya [22], Kaya and De Souza [23] have studied barbells and dumbbells and the related convex particles, i.e. capped cylinders. They calculated the corresponding form factors. Senesi and Lee [24] have indirectly studied non convex bodies. They presented a general method to calculate the scattering functions of polyhedra. These are calculated by breaking the body into sets of pieces. This work included the calculation of concave bodies. Ciccariello et al. [25a-b] have considered the small-angle scattering from anisotropic samples and have found a simple expression between the scattering intensity and the absolute values of Gaussian curvature at particular surface points. They show that this equation is also valid for non convex particles. This corpus of work emphasizes the links between scattering theory and stochastic geometry, integral geometry and differential geometry.

Among the particle shapes observed in suspensions during a precipitation or crystallization process, small clusters of spherical particles are often present [19]. The contact between the two spheres in the cluster can be a single point or a neck due to sintering. This type of particle is one of the simplest cases of non convex bodies. Therefore, in this paper, we explore the properties of their CLD's and the relationship between CLD and convexity. Due to the complexity and difficulty of exact analytical calculation, CLD's will be obtained from MonteCarlo Simulations (MCS).

The rest of the paper is organized as follows: section two introduces the algorithms used for MCS. The data issued from MCS for some non convex bodies are presented and discussed in the section three, followed by a conclusion and perspectives for future work in section four. 


\section{Chord length distributions by Monte-Carlo Simulations}

Our work focuses on OCD calculations with 3D uniform flow of lines.

Note: throughout the paper and the literature, the chord length distribution (density) is written $D_{l}(l)$ where $0 \leq l \leq l_{\max } . D_{l}(l) d l$ is the number of chords within the $l$-range $[l, l+d l] . D_{l}(l)$ is usually presented as normalized, i.e $\int_{0}^{l_{\max }} D_{l}(l) d l=1$.

In this article three kinds of particles are considered and compared, that are bodies of revolution along the x-axis. They are composed of spheres or parts of a sphere. The centres of spheres are symmetrically located along the x-axis. The origin of the coordinate system is the symmetry centre of the particle. The three particles are:

- $\quad$ sphere with radius $\mathrm{R}_{1}$ (figure 1a)

- cluster of two overlapping spheres (figure $1 \mathrm{~b}$ ); the radius of spheres is $\mathrm{R}_{1}$ and the distance between the two centres is denoted $\delta$. This type of cluster will be designated dumbbell [19]. A particular case is the cluster of two touching spheres: $\delta=2 R_{1}$.

- a cluster of two touching spheres where the neck (or overlap) is partially filled with matter (figure 1c); the radius of spheres is $\mathrm{R}_{1}$ and the upper boundary of the neck is a part of a torus with a minor radius $\mathrm{R}_{2}$; as the torus is tangentially linked to the spheres, the major radius $\mathrm{R}_{3}$ obeys the expression $R_{3}=\sqrt{R_{2}^{2}+2 R_{2} R_{1}}$. This type of cluster will be called a diabolo. An example is the convex body corresponding to $R_{2} \rightarrow \infty$, i.e. a capsule. The cluster of two touching spheres corresponds to $R_{2} \rightarrow 0$.

A MCS software was employed to generate an isotropic uniform random line across the geometric object, and to collect the chord length segments. The same framework for the Monte Carlo Simulations (MCS) of the different particle shapes has been used.

All the distances are normalized by $\mathrm{R}_{1}$ (then, $\mathrm{R}_{1}=1$ ). Consider a sphere with radius 2 and its centre located at the origin. The way used to define the random straight line is the following: 
A direction and a point belonging to the plane orthogonal to that direction and tangent to the sphere are considered. The coordinate system of the plane is composed of the point of tangency and the vectors from the usual spherical coordinate system. The line will be defined by the two angles, polar $\theta$ and azimuthal $\phi$, and the two coordinates $\mathrm{x}_{\mathrm{P}}$, $\mathrm{yP}_{\mathrm{P}}$ of the point in the plane. Four random numbers [26] are chosen for the values of the variables $\cos \theta, \phi, x_{P}$ and $y_{P}$. The line intersects the sphere at two points denoted $\mathrm{M}_{1}$ and $\mathrm{M}_{2}$. This algorithm is known to provide a translation and rotation invariant density [27].

Depending on the particle, the straight line between $\mathrm{M}_{1}$ and $\mathrm{M}_{2}$ may intersect the particle 0,2 , 4 or 6 times. The intersection points will be analytically determined and the corresponding distances calculated. The details of these calculations based on the analytic geometry are reported in appendix A (for the sphere), Appendix B (for the dumbbell) and Appendix C (for the diabolo).

The MC sampling distribution may be visually represented as a discrete probability histogram. The chord length between zero and the maximal possible length is divided on mbins with the equal size of $\Delta \mathrm{l}$. The chord lengths are normalized by the radius of spheres $(0 \leq l \leq 4)$. The value of the $l$-step $\Delta 1$ is 0.01 . All simulation runs have been carried out by generating $\mathrm{NT}=10^{8}$ unbiased random lines. Only a smaller number $N l$ lines cross the body. The sampling error is smaller than $10^{-4}$.

We have compared our new MC algorithm with the previous [19] and with analytical calculations (sphere, ellipsoid, cylinder and dumbbells with various inter-centre distance $\delta$ ) coming from the same paper.

As the interaction between the random line flow and different bodies (sphere, dumbbell and diabolo) are compared by using the same process, we may present the CLD as nonnormalized:

$D\left(l_{i}\right)=N_{i} /(N T \Delta l)$ 
where $N_{i}$ is the number of chord with the length $l_{i}$.

As already mentioned, the CLD is usually presented as normalized:

$D_{l}\left(l_{i}\right)=N_{i} /(N l \Delta l)$

The random variable chord length, denoted by $l$, was made dimensionless by dividing the chord value by the radius $R_{l}$ of the sphere in Eqs. 1-2.

The number $N l$ depends on $N T$ and the characteristics of the body following:

$$
N l / N T=\langle S p\rangle / S_{T}
$$

where $\langle S p\rangle$ and $S_{T}$ are the averaged projected area of the body and the area of the sphere with radius 2. Table 1 reports the values of $N l / N T$ for dumbbells with various $\delta$-values and for diabolos with various $R_{2}$ - values. The validity of Eq. 3 has been successfully verified.

These two definitions of the CLD will be used in the section three.

\section{Results and discussion}

\subsection{Distribution density function}

The figures $2 \mathrm{a}-\mathrm{b}$ represent the CLDs of dumbbells with various inter-centre values $\delta$ whereas the figures $3 a-b$ represent the CLDs of diabolos with various neck sizes expressed as $R_{2}$. The CLD's of two touching spheres [18] and dumbbells [19] are analytically known; the comparison between analytical expressions and MCS shows a very strong agreement.

The main contribution to the CLD's for the two shapes comes from the constitutive spheres $(0<l<2)$. The contribution of large chord length $(l>2)$ is always minor.

In the two cases a discontinuity for $l=2$ is observed. However this is more pronounced for the dumbbell. This seems related to the details of the concavity of the body: the curvature varies smoothly for the diabolo whereas there is a discontinuity of the curvature for the dumbbell. 
A parameter for quantifying the non convexity of the two objects was needed. Therefore we chose the depth $P_{C}$ of the concavity divided by the radius $R_{l}$ of the sphere:

$P_{C}=1-\sqrt{1-\delta^{2} / 4} \quad$ for the dumbbell

$P_{C}=1+R_{2}-\sqrt{R_{2}^{2}+2 R_{2}} \quad$ for the diabolo

By comparing the CLD's of a sphere, a dumbbell and a diabolo, the following features are observed:

- The value of the distribution density $D_{l}$ is equal to zero at $l=0$ for the three shapes. The slope of the tangent line at $1=0$ is $0.44 \pm 0.01$ for the dumbbells within the $\delta$-range studied. The slope of the tangent line at $1=0$ is $0.38 \pm 0.06$ for the diabolos within the $\mathrm{R}_{2}$-range studied.

- A discontinuity of the derivative of the CLD $D_{l}$ at $l=l_{\max }$ is observed for dumbbells and diabolos whereas $D_{l}\left(l_{\max }\right)=0$.

- A discontinuity of the derivative of the CLD $D_{l}$ in the range $[0 ; 2]$ of chord length values. This appears at a length value denoted $l_{1}$ and the discontinuity of the curve slope is characterized by an angle jump denoted $\theta_{1}$. The angle jump is positive in the case of the dumbbell whereas it is negative in the case of the diabolo.

- A discontinuity of the CLD $D_{l}$ at $1=2$, denoted $\Delta 2$.

- The curve slope when $l \rightarrow 2^{+}$, also corresponding to a slope discontinuity. The curve slope is characterized by the angle $\theta_{2}$ between the tangent line and the x-axis.

- The curvature of the curve when $l>2$. The curvature is negative in the case of the dumbbell whereas it is positive in the case of the diabolo.

All the parameters $l_{1}, \theta_{1}, \Delta 2, \theta_{2}$ depend on the non convexity parameter $P_{C}$. Now, let us examine the corresponding empirical relations. Unfortunately, the Monte-Carlo simulations are not precise enough to determine $\theta_{1}$ and $\theta_{2}$ with accuracy. 
Whatever the shape of the objects, there exists a simple expression between $l_{1}$ and $P_{C}$ :

$$
l_{1} \approx 2\left(1-P_{C}\right)
$$

This shows that $l_{l}$ corresponds to the chord crossing the concavity perpendicularly to the line joining the sphere centres. The comparison of the angle jumps $\theta_{1}$ (calculated from $D_{l}$ ) only shows qualitative trends:

- The angle jump is within the range $[0 ; \pi / 2]$ in the case of the dumbbell. It is a decreasing function of $P_{C}$.

- The angle jump is within the range $[-\pi ; 0]$ in the case of the diabolo. It is an increasing function of $P_{C}$.

Whatever the shape of the objects, the relation between $\Delta 2$ (calculated from $D_{l}$ ) and $P_{C}$ is:

$$
\Delta 2 \approx\left(0.2+3 P_{C}\right) \frac{S_{T}}{l_{\max }\left\langle S_{P}\right\rangle}
$$

The comparison of the $\theta_{2}$ angles (calculated from $D_{l}$ ) only shows rough trends:

- In the case of the dumbbell $\theta_{2}$ is within the range $[-\pi / 2 ; 0]$. It is a strongly increasing function of $P_{C}$.

- In the case of the diabolo $\theta_{2}$ is within the range $[-\pi / 2 ; 0]$. It is a weakly increasing function of $P_{C}$.

Most published studies concern convex bodies. Any convex body may be considered as an equivalent spheroid or ellipsoid, i.e. a body with a smooth shape, modified by specific geometrical features. These features are important for the shape of the CLD curve. They consist in flat faces as crystal facets, parallel (flat or curved) surfaces, parallel tangent planes, edges and corners. They correspond to discontinuities of the distribution density or its derivative. Here are the main results from the literature: 
i) Begin by investigating the effect of the curvature on CLD. For a convex body with a smooth surface the series expansion of CLD around $l=0$ does not contain even terms. The first order term is an explicit function of the mean principal curvatures of the body [28, 29]:

$D_{l}(l)=D_{1} l+D_{3} l^{3}+\ldots$

With $D_{1}=\frac{1}{2}\left\langle k_{1} k_{2}\right\rangle+\frac{3}{16}\left\langle\left(k_{1}-k_{2}\right)^{2}\right\rangle$

$k_{1}$ and $k_{2}$ are the principal curvatures at some point on the surface of the body. $<>$ is the averaging over all the surface area. The terms with order higher than 1 have no tractable expression. $D_{3}$ is equal to zero for an ellipsoid.

ii) The presence of edges leads to additional terms for $D_{l}(l)$ at $l=0$. Ciccariello et al. [3031] and Sobry et al. [32-33] have shown that $D_{l}(0)$ is a simple function of the dihedral angle and edge length. All the edges contribute to $D_{l}(0)$. Edges (and corners) also contribute to $d D_{l}(l) /\left.d l\right|_{l=0}[34]$. However, it seems difficult to systemize this contribution. iii) Chords intersect the surface at two points in a convex body; some chords are perpendicular to the tangent planes at the two end points and thus the planes are parallel. These chords are termed extremal chords. For instance, spheroids [35] have two extremal chords: one for $l=2 a$ and the other for $l=2 b, a$ and $b$ being semi-axes. A discontinuity of $d D_{l}(l) / d l$ will occur at these values. Wu and Schmidt [36] have investigated the properties of $D_{l}(l)$ when the chord is in the neighbourhood of an extremal chord. They give expressions for $D_{l}(l)$ around the extremal chord values denoted $L$. The authors also show that $D_{l}(l)$ is continuous for $l=L$ whereas $d D_{l}(l) / d l$ is not.

iv) Ciccariello [30, 37, 38] generalized the work of $\mathrm{Wu}$ and Schmidt: he considered the property of parallelism between some parts of body boundaries. He studied the case where 
the locus of the extremal chord ends is a surface. For instance, this surface is a sphere for $L=l=2 R$ if the particle is a sphere with radius $R$. One can show that $D_{l}(l)$ becomes discontinuous for this chord length value. If the parallelism occurs between two partial surfaces of the body, a discontinuity of $D_{l}(l)$ occurs at $l=L, L$ being the distance between the two parallel surfaces. The contribution of the parallelism to $D_{l}(l)$ for $l \rightarrow L$ is given by Ciccariello.

Diabolos and dumbbells are rounded bodies with curved surfaces and tangent planes that are both parallel, but without edges. The circular junction between the two spheres of the dumbbell cannot be considered as an edge. The edges considered in the literature are the ones occurring in convex crystals with facets or finite cylinders. In this case, the grazing lines cross the edge more frequently than the smooth surface. Therefore, $D_{l}(0)$ is not equal to zero. In the case of the dumbbell, the lines crossing the circular edge do not correspond to $l=0$. Rather, the lines crossing the circular edge correspond to a large range of chord length values $(\neq 0)$. Hence, the value of the distribution density at $l=0$ is equal to zero. For a smooth convex body the slope $D_{l}$ of $D_{l}(l)$ at $l=0$ depends on the mean values of principal curvatures. The mean values are obtained after averaging over all the surface area. One may expect that the Eqs.7a-b hold only for the convex boundaries of non convex bodies. However, some lines cross the body twice with one segment length close to 0 and the other one having a finite value: these (OCD) lines do not contribute to $D_{1}$. Thus, in the case of OCD, it seems difficult to interpret further the $D_{1}$ data. Nevertheless, one observes that $D_{1}$ is a very weak function of $\delta$ or $R_{2}$; the corresponding averaged value is slightly smaller than 0.5 which is the corresponding value for a sphere.

Diabolos and dumbbells contain extremal chords at several values of the chord length; we will investigate the corresponding situations: 
- $L=l_{l}$ : corresponds to the diameter of the circle that is the intersection of the object and the symmetry plane perpendicular to the axis of revolution. This is similar to the intersection of a prolate spheroid with a plane containing the two minor axes [35]. One observes in the two cases a discontinuity of the derivative of the distribution density. This is due to the occurrence of a set of tangent planes at each end point of the diameter. However, such tangent planes are difficult to define for dumbbells. The theory of $\mathrm{Wu}$ and Schmidt does not apply to these non convex bodies.

- $\quad L=2$ : the corresponding chord is the diameter of each constituting spheres. The locus of the chord ends is a part of the sphere surfaces. Following Ciccariello [30, 37, 38], a discontinuity of the CLD density is expected:

$$
\Delta 2=-2 S_{i} /(L S)
$$

where $S$ and $S_{i}$ are respectively the surface area of the body and the surface area swept by a line passing through the centre of each sphere and crossing only the surface of this sphere.

The corresponding expressions for dumbbells are:

$$
\begin{array}{llll}
\Delta 2=-2 \frac{\sqrt{1-\left(R_{1} / \delta\right)^{2}}}{R_{1}+\delta / 2} & \text { if } & \delta / R_{1}>\sqrt{2} \\
\Delta 2=-2 \frac{\delta /\left(2 R_{1}\right)}{R_{1}+\delta / 2} & \text { if } & \delta / R_{1}<\sqrt{2}
\end{array}
$$

Whereas for the diabolos:

$$
\Delta 2=-2 \frac{R_{1} \sin \alpha}{R_{1}^{2}+\left(R_{1}^{2}-R_{2}^{2}\right) \sin \alpha-R_{2} R_{3} \alpha} \quad \text { with } \quad \sin \alpha=R_{1} /\left(R_{1}+R_{2}\right)
$$

The Monte-Carlo simulations are in agreement within 5\% with these expressions. The accuracy given is expected because one has to take into account the discretization of the chord length domain in the distribution curve coming from MC simulations. 
Hence, the expression of Ciccariello works for non convex body considering that the expressions are applied to the convex sub-surface of this body.

- $L=l_{\max }$ : the corresponding chord is perpendicular to the tangent planes at the two end points; Following Wu and Schmidt [36], the distribution density obeys the relation:

$$
D_{l}(l)=\frac{8 \pi R_{1}^{2}\left(l_{\max }-l\right)}{l_{\max }\left(l_{\max }-2 R_{1}\right) S} \quad l<l_{\max }
$$

The corresponding expression for dumbbells is:

$$
D_{l}(l)=\frac{4 R_{1}\left(\delta+2 R_{1}-l\right)}{\delta\left(\delta+2 R_{1}\right)^{2}}
$$

Whereas for the diabolos:

$$
D_{l}(l)=\frac{\left(4 R_{1}-l\right)}{4 R_{1}^{2}(1+\sin \alpha)+4 R_{2}\left(R_{3} \alpha-R_{2} \sin \alpha\right)}
$$

The simulations are in agreement within $5 \%$ for the dumbbells and $1 \%$ for the diabolos. Therefore, the expression of $\mathrm{Wu}$ and Schmidt works for the non convex bodies considering that the expressions are applied to the convex sub-surface of these bodies.

The angle $\theta 2$ is related to the convexity of the particle. Surprisingly, the shape of the CLD when $l \rightarrow 2^{+}$looks like the non convex part of the particle shape. The chords with $l>2(l$ close to 2) correspond mainly to lines including the diameter of a given sphere. In the case of the diabolo, the other part of the line crosses the neck between the two spheres; hence a correspondence between CLD shape and the local curvature of the particle is expected. In the case of dumbbells, the other part of the line belongs to the other sphere; the CLD shape when $l \rightarrow 2^{+}$depends on the other sphere. However in the two cases the calculation of the CLD when $l \rightarrow 2^{+}$has proven difficult. As a consequence, the angle $\theta 2$ is not a simple function of the geometrical parameters of the particle.

\subsection{Distribution moments}


Moments of CLD are related to some integral characteristics, e.g. surface area, volume, mean curvatures, of the body. It is well known that the first and the fourth order moments can be expressed as a function of $S$ and $V$ for convex bodies. It has been shown [15] from the study of a special category of non convex bodies that the fourth order moment cannot be expressed as a function of simple geometric properties. Thus, the following discussion focuses to the first order moment that is the mean chord length. The mean chord length of a convex body is related to $V$ and $S$ by means of the Cauchy formula:

$\bar{l}=4 V / S$

Mazzolo et al. [39] cite the book of Solomon [5] where this author showed that a modified Cauchy equation holds for a non convex body:

$\overline{l_{O C D}}=4 V / S^{*}$

Where $S^{*}$ is the surface area of the convex hull of the body.

Mazzolo et al. [39] calculates $\overline{l_{M C D}}$ for various non convex bodies. Monte Carlo simulations are used to validate the expressions given for $\overline{l_{M C D}}$ (Table I in [39]). The figure 7 (in [39]) corresponds to the CLD for two touching spheres in the MCD case. Hence, there is no confirmation in [39] that the modified Cauchy (OCD) equation holds for non convex bodies. Moreover, Gille ([20], pages 125-126) mentions that the mean chord length for any two touching spheres obeys the relation:

$\overline{l_{O C D}}=1.44236 R_{1}$ and $\overline{l_{M C D}}=1.333 R_{1}$

This is in agreement with our calculations. On the other hand, equation 15 leads to $\overline{l_{O C D}}=1.333 R_{1}$. Hence, equation 15 is not valid for the particle class studied herein.

Additional calculations lead to the following results:

- The equation $\overline{l_{M C D}}=4 V / S$ has been tested and found to hold for dumbbells with various $\delta$-values. 
- In addition, equation 15 has been tested for both dumbbells and diabolos. We have compared the mean chord lengths (OCD) coming from Monte-Carlo simulations $l_{M C}$ and from the equation 15 , named $l_{t h}\left(=\overline{l_{O C D}}\right)$. Figures $4 \mathrm{a}$ and $4 \mathrm{~b}$ represent the ratio $l_{\text {th }} / l_{M C}$ versus $\delta$ for dumbbells and versus $R_{2}$ for diabolos respectively (red circles). We conclude that the equation 15 does not hold in these cases. Calculating $l_{t h} / l_{M C}$ with $l_{t h}=4 \mathrm{~V} / \mathrm{S}$ also shows a slight deviation from 1 (the green pluses on figures $4 \mathrm{a}$ b). On the other hand, the expression $l_{t h}=V /\left\langle S_{P}\right\rangle$ leads to a very good agreement with $l_{M C} \cdot\left\langle S_{P}\right\rangle$ is the average projected area over all the orientations of the body. The analytical expression for $\left\langle S_{P}\right\rangle$ is in the case of the dumbbells:

$$
\left\langle S_{P}\right\rangle=2 R_{1}^{2} \int_{0}^{\pi / 2} \sin \vartheta\left[\pi-a \cos (u \sin \vartheta)+2 u \sin \vartheta \sqrt{1-u^{2} \sin ^{2} \vartheta}\right] d \vartheta
$$

with $u=\delta /\left(2 R_{1}\right)$

Searching a relation between the distribution moments and geometrical quantities of the body and its convex hull is natural in the field of stochastic geometry. Hence, the empirical expression $l_{t h}=4\left(V+\left(V^{*}-V\right) / 3\right) / S^{*}$ was considered. $V^{*}$ is the volume of the convex hull of the body. This means that the volume to be considered is the sum of the matter volume and one-third of the hollow volume inside the convex hull. This is correct at least for these two different particle shapes. It should be underlined that the non convex part of the particle is not a closed porosity as studied by Mazzolo et al.[15, 39]. Our result should be read as a numerical experiment only valid for these two cases. This seems an interesting approximation knowing that $V$ and $S$ for the convex hulls can be much easier calculated than those of non convex particles. 


\section{3. (OCD) CLD and scattering properties}

Rayleigh-Debye-Gans (RDG) theory is a scattering theory for light that is valid for optically soft material [2]. It may be applied to small soft particles with a small phase shift, namely: $2 \pi d / \lambda|m-1|<<1 . d, \lambda, k=2 \pi / \lambda, m$ are respectively the characteristic length of the scatterer, wavelength, modulus of the wave vector, ratio of the refractive indices of the particle and surrounding medium. Nota bene: SAS methods are based on RDG theory.

Within the RDG approximation, the differential scattering cross section per unit incident intensity (unpolarized light) is related to the amplitude form factor $R$. Considering that the object (scatterer) can randomly orientate, this optical property is thus obtained after calculating an average value over all the orientations of the object:

$$
\left\langle C_{\text {diff sca }}\right\rangle=\frac{k^{4} V^{2}}{4 \pi^{2}}|m-1|^{2}\left\langle|R|^{2}\right\rangle \frac{1+\cos ^{2} \theta}{2}
$$

with

$$
\left\langle|R|^{2}\right\rangle=\int_{0}^{l_{\max }} \frac{\sin (q r)}{q r} D_{P}(r) d r
$$

$\theta$ and $q(q=2 k \sin (\theta / 2))$ are the scattering angle and modulus of the scattering vector, respectively. $D_{P}$ is the distance distribution function. The distance $r$ is the length of the line segment connecting two points inside the scatterer. $D_{P}$ is proportional to $r^{2} \gamma(r)$ where $\gamma(r)$ is the correlation function.

Following the theory of stochastic geometry [6], there exists a relation between the two normalized distributions $D_{P}$ and $D_{l}$ for a convex particle:

$$
D_{P}(r)=4 \pi r^{2} \int_{r}^{l_{\max }} D_{l}(l)(l-r) d l /(V \bar{l})
$$


By introducing Eq.19 into Eq.18, one expresses $\left\langle|R|^{2}\right\rangle$ and the differential scattering cross section as a function of the CLD [9]:

$$
\left\langle|R|^{2}\right\rangle=\frac{4 \pi}{\bar{l} V q^{4}}\left[2-\int_{0}^{l_{\max }}(q l \sin (q l)+2 \cos (q l)) D_{l}(l) d l\right]
$$

The equivalence between Eqs. 18 and 20 is strictly valid for convex particles [40]. We will compare the data issued from Eqs.18 and 20 for non convex particles. The figure 5 represents $\left\langle|R|^{2}\right\rangle$ as a function of $q R_{1}$ for a diabolo with various $R_{2}$ values. The diabolo with $R_{2}=100$ looks very much like a convex body (capsule); as expected the two data sets fit. The diabolo with $R_{2}=0.01$ is similar to a dumbbell with $\delta=2 R_{1}$ : the two data sets are surprisingly close to each other. This result is also verified for $R_{2}=1$. One may conclude that Eq.20 using CLD (OCD) represents a zeroth-order approximation for the calculation of scattering properties.

Numerical experiments show that $\left\langle|R|^{2}\right\rangle$ is much more sensitive to numerical errors on $D_{P}$ than on $D_{l}$. Hence it will be better to use $D_{l}$ instead of $D_{P}$.

\section{Conclusion}

The CLD of non convex bodies contains specific features which could lead to an easier identification of their shape. These are discontinuities of the distribution function or of its derivative. Such discontinuities of CLD are known in the literature. They concern several convex bodies. In our case of dumbbells and diabolos, the CLD is the superposition of the CLD of a sphere (or a capsule) and of the specific features related to some local non convexity. These features depend on a single parameter that characterizes the non convexity. Our results confirm some mathematical properties of CLD and its moments. Theses results deal with the convex parts of concave bodies. Specific features related to non convexity have 
been also investigated. Thus we are able to qualitatively reconstruct the CLD of such concave bodies, as this has been recently performed for convex bodies [41]. Furthermore, the use of CLD-OCD for the calculation of the scattering properties provides a correct estimate of the differential scattering cross section.

The results depend on the shape of the convex hull of the body which is the main contribution to the CLD. If the body shape is the geometrical superposition of the convex hull and cavities located on its surface, it may be easy to extend the results to such a body. This will be investigated in the future. This study has centred on rounded bodies. It would be interesting to investigate the influence of non convexity on the CLD of clusters composed of facetted crystals in up coming research.

\section{Acknowledgment:}

The author wishes to thank Pr. Chris Yukna for help in proofreading.

\section{References}

[1] A. Guinier and G. Fournet, Small-Angle scattering of X-rays, Wiley, New York, 1955

[2] H.C. Van de Hulst, Light scattering by small particles, John Wiley, NewYork, 1957.

[3] M. Li and D. Wilkinson, "Determination of non-spherical particle size distribution from chord length measurements. Part 1: theoretical analysis", Chemical Engineering Science, 60(2005)3251-3265.

[4] A.A. Kokhanovsky, Optics of light scattering media, Springer, Berlin, 2001

[5] H. Solomon, Geometric probability, n²8, 1978, SIAM, Philadelphia

[6] L.A. Santalo, Integral geometry and geometric probability, 2004, Cambridge Mathematical Library 
[7] R.V. Ambartzumian, Combinatorial integral geometry with applications to mathematical stereology, 1982, J. Wiley, Chichester, N.Y.

[8] V.K. Ohanyan and N.G. Aharonyan "Tomography of bounded convex domains" International Journal of Mathematical Science Education 2(2009)1-12

[9] W. Gille, "The small-angle scattering correlation function of the hemisphere", Computational Materials Science, 1999, 15: p. 449-454

[10] W. Gille "Chord length distributions of infinitely long geometric figures" Powder Technology 123(2002)292-298

[11] W. Gille "Chord length distribution density of a triangular rod" Computational Materials Science 22(2001)151-154

[12] R. Garcia-Pelayo "Distribution of distance in the spheroid", J. of Physics A: Mathematical and General, 38(2005)3475-3482

[13] H.S. Sukiasian "Three-dimensional Pleijel identity and its application" Izvestiya Natsionalnoi Akademii Nauk Armenii Matematika 38(2003)53-69

[14] S. Ciccariello, "The chord-length probability density of the regular octahedron", arXiv: $1401.7950 \mathrm{v} 2$

[15] A. Mazzolo, B. Rooesslinger, C.M. Diop, "On the properties of the chord length distribution, from integral geometry to reactor physics", Annals of Nuclear Energy 30(2003)1391-1400

[16] W. Gille "Chord length distribution density of an infinitely long circular hollow cylinder" Mathematical and computer modelling 34(2001)423-431

[17] A. Yu. Vlasov "Signed chord length distribution" arXiv:0711.4734v3 [math-ph] 17 Dec 2007

[18] F. Gruy and S. Jacquier, "The chord length distribution of a two-sphere aggregate", Computational Materials Science 44(2008)218-223 
[19] F. Gruy and S.H. Suh, "The chord length distribution of a dumbbell shaped aggregate: analytical expression”, Powder Technology 253(2014)207-215

[20] W. Gille, "Particle and particle systems characterization: small-angle scattering applications", CRC Press, 2014, New York

[21] W. Gille "The small angle scattering correlation function of two infinitely long parallel circular cylinders” Computational Materials Science 20(2001)181-195

[22] H. Kaya, "Scattering from cylinders with globular end-caps", J. Applied Crystallography $37(2004) 223-230$

[23] H. Kaya and N-R de Souza, "Scattering from capped cylinders. Addendum", J. Appl. Cryst. 37(2004)508-509

[24] A. Senesi, B. Lee, "Scattering functions of polyhedra”, J. Appl. Cryst. 48(2015)565-577

[25a] S. Ciccariello, J-M Schneider, B. Schönfeld, G. Kostorz, “Generalization of Porod's law of small-angle scattering to anisotropic samples“, Europhys. Lett. 50(2000)601-607

[25b] J.-M. Schneider, S. Ciccariello, B. Schönfeld and G. Kostorz, The asymptotic leading term of anisotropic small-angle scattering intensities. II. Non-convex particles, Acta Cryst A58(2002)221-231

[26] A. Mazzolo and B. Roesslinger, "Monte Carlo simulation of the chord length distribution function across convex bodies, non convex bodies and random media", Monte Carlo Methods and Applications, 10(2004)443-454

[27] E.T. Jaynes, “The well posed problem”, Papers on Probability, Statistics and Statistical Physics, R.D. Rosencrantz Ed, D. Reidel, Dordrecht (1983)133-148

28] R. Kirste \& G. Porod, "Röntgenkleinwinkelstreuung an Kolloiden Systemen. Asymptotisches Verhalten der Streukurven“, Kolloid Zeitschrift \& Zeitschrift für Polymere 184(1962)1-7 
[29] H. Wu and P.W. Schmidt, "Intersect distributions and small-angle X-ray scattering theory" J. Appl. Cryst. 4(1971)224-231

[30] S. Ciccariello and A. Benedetti, "Parametrizations of scattering intensities and values of the angularities and of the interphase surfaces for three-component amorphous samples", J. Appl. Cryst. 18(1985)219-229

[31] S. Ciccariello, "Edge contributions to the Kirste-Porod formula: the spherical segment case", Acta Cryst. A49(1993)750-755

[32] R. Sobry, J. Ledent and F. Fontaine, "Application of an extended Porod law to the study of the ionic aggregates in telechelic ionomers", J. Appl. Cryst. 24(1991)516-525

[33] R. Sobry, F. Fontaine and J. Ledent, "Extension of Kirste-Porod's law in the case of angulous interfaces”, J. Appl. Cryst. 27(1994)482-491

[34] S. Ciccariello and R. Sobry, "the vertex contribution to the Kirste-Porod term", Acta Cryst. A51 (1995)60-69

[35] A.M. Kellerer, Chord Length Distributions and related quantities for spheroids, Radiation Research, 98(1984)425-437

[36] H. Wu and P.W. Schmidt, "The relation between the particle shape and the outer part of the small-angle X-ray scattering curve” J. Appl. Cryst. 7(1974)131-146

[37] S. Ciccariello, "Deviations from the Porod law due to parallel equidistant interfaces", Acta Cryst. A41(1985)560-568

[38] S. Ciccariello, "The leading asymptotic term of the small-angle intensities scattered by some idealized systems", J. Appl. Cryst. 24(1991)509-515

[39] A. Mazzolo, B. Roesslinger, W. Gille, "Properties of chord length distributions of non convex bodies", Journal of Mathematical Physics, 44(2003),6195-6209

[40] S. Hansen, "Estimation of chord length distributions from small-angle scattering using indirect Fourier transformation”, J. Applied Crystallography 36(2003)1190-1196 
[41] F. Gruy, "Stochastic geometry for electromagnetic scattering modelling", Proceedings of PIERS 2015, 1248-1251, Prague 
Appendix A: Algorithm for a sphere

The radius of the sphere is equal to 1 and its centre has a x-coordinate denoted $a$. We consider the intersection between the sphere and a straight line as defined by the vector relation: $\overrightarrow{M_{1} M}=\lambda \overrightarrow{M_{1} M_{2}}$ where $\lambda$ is a real number to determine (Figure A.1). There are 0 or 2 intersection points. The coordinates of $\mathrm{M}_{\mathrm{i}}$ points $(i=1,2)$ are denoted by $x_{i}, y_{i}, z_{i}$.

The coordinates of the intersection points obey the system of algebraic equations:

$$
\left\{\begin{array}{l}
(x-a)^{2}+y^{2}+z^{2}=1 \\
x-x_{1}=\lambda\left(x_{2}-x_{1}\right) \\
y-y_{1}=\lambda\left(y_{2}-y_{1}\right) \\
z-z_{1}=\lambda\left(z_{2}-z_{1}\right)
\end{array}\right.
$$

Thus, $\lambda$ is a solution of the polynomial:

$$
A \lambda^{2}+2 B(a) \lambda+C(a)=0
$$

with

$$
\begin{aligned}
& A=\left(x_{2}-x_{1}\right)^{2}+\left(y_{2}-y_{1}\right)^{2}+\left(z_{2}-z_{1}\right)^{2} \\
& B(a)=\left(x_{2}-x_{1}\right) x_{1}+\left(y_{2}-y_{1}\right) y_{1}+\left(z_{2}-z_{1}\right) z_{1}-a\left(x_{2}-x_{1}\right) \\
& C(a)=x_{1}^{2}+y_{1}^{2}+z_{1}^{2}-2 a x_{1}+a^{2}-1
\end{aligned}
$$

If $B^{2}-A C<0$ there is no intersection point

If $B^{2}-A C>0$ there are two intersection points characterized by the real numbers $\lambda_{+}$and $\lambda_{-}$:

$$
\lambda_{ \pm}=\left(-B \pm \sqrt{B^{2}-A C}\right) / A
$$

The chord length will be:

$$
l=\left|\lambda_{+}-\lambda_{-}\right| \sqrt{A}
$$

Appendix B: Algorithm for a dumbbell 
Let us consider the projection $\mathrm{P}_{\mathrm{j}}$ of the two sphere centres $\mathrm{O}_{\mathrm{j}}$ on the plane perpendicular to $\overrightarrow{M_{1} M_{2}}$ and containing $\mathrm{M}_{1}$ (Figure B.1) The corresponding coordinates are:

$x_{p}(a)=a+\left(x_{2}-x_{1}\right) B(a) / A$

$y_{p}(a)=\left(y_{2}-y_{1}\right) B(a) / A$

$z_{p}(a)=\left(z_{2}-z_{1}\right) B(a) / A$

Then, let us define the quantities (Figures B.1 and B.2):

$$
\begin{aligned}
& Q_{1}=Q(-\delta / 2) \\
& Q_{2}=Q(\delta / 2)
\end{aligned}
$$

With

$$
Q(a)=1-M_{1} P_{j}^{2}=1-\left(x_{p}(a)-x_{1}\right)^{2}-\left(y_{p}(a)-y_{1}\right)^{2}-\left(z_{p}(a)-z_{1}\right)^{2}
$$

and

$Q_{12}=P_{1} P_{2}^{2}=\left(x_{p}(\delta / 2)-x_{p}(-\delta / 2)\right)^{2}+\left(y_{p}(\delta / 2)-y_{p}(-\delta / 2)\right)^{2}+\left(z_{p}(\delta / 2)-z_{p}(-\delta / 2)\right)^{2}$

The chord length $l$ is a function of $Q_{1}, Q_{2}, Q_{12}$ and $\delta$.

Then,

If $Q_{1}<0$ and $Q_{2}<0$ then $l=0$

If $Q_{1}>0$ and $Q_{2}<0$ then $l=2 \sqrt{Q_{1}}$

If $Q_{1}<0$ and $Q_{2}>0$ then $l=2 \sqrt{Q_{2}}$

If $Q_{1}>0$ and $Q_{2}>0$ then

$$
\left\{\begin{array}{l}
d=\sqrt{Q_{1}}+\sqrt{Q_{2}}+\left(\delta^{2}-Q_{12}\right)^{1 / 2} \\
D=2 \sqrt{Q_{1}}+2 \sqrt{Q_{2}} \\
S=\sup \left\{2 \sqrt{Q_{1}}, 2 \sqrt{Q_{2}}\right\}
\end{array}\right.
$$

$$
\text { If } D>d \text { then } l=\sup \{S, d\}
$$


$2 \sqrt{Q_{i}}$ is the length of the chord in the sphere $i . d$ is the distance between the two end points of the line crossing the two spheres.

Appendix C: Algorithm for a diabolo

Consider the intersection of the straight line with the two spheres and the torus fragment. Ns $(=0,2$ or 4$)$ is the total number of intersection points with the spheres. $\mathrm{Nt}(=0,1,2)$ is the number of intersection points with the torus fragment.

The intersection with each sphere, when and if it occurs, is characterized by the $\lambda$ values:

$$
\lambda_{ \pm}=\left(-B \pm \sqrt{B^{2}-A C}\right) / A
$$

The possible intersection with the torus is characterized by the $\lambda$ values that are the roots of a quartic polynomial:

$a_{4} \lambda^{4}+a_{3} \lambda^{3}+a_{2} \lambda^{2}+a_{1} \lambda+a_{0}=0$

With

$$
\begin{aligned}
& a_{0}=\left(C(0)+1-R_{3}^{2}-R_{2}^{2}\right)^{2}+4 R_{3}^{2}\left(x_{1}^{2}-R_{2}^{2}\right) \\
& a_{1}=4 B(0)\left(C(0)+1-R_{3}^{2}-R_{2}^{2}\right)+8 R_{3}^{2}\left(x_{2}-x_{1}\right) x_{1} \\
& a_{2}=4 B(0)^{2}+2 A\left(C(0)+1-R_{3}^{2}-R_{2}^{2}\right)+4 R_{3}^{2}\left(x_{2}-x_{1}\right)^{2} \\
& a_{3}=4 A B(0) \\
& a_{4}=A^{2}
\end{aligned}
$$

As only a part of the torus is considered, select the $\lambda$-values that obey the inequality:

$$
\left(y_{1}+\lambda\left(y_{2}-y_{1}\right)\right)^{2}+\left(z_{1}+\lambda\left(z_{2}-z_{1}\right)\right)^{2}<R_{3}^{2} /\left(1+R_{2}\right)^{2}
$$

With this condition, there are 0,1 or 2 real values for $\lambda$.

Then, all the real $\lambda$-values (from the two spheres and torus) are classified in increasing order. One gets a series of $\lambda$-values $\left(\lambda_{1}<\lambda_{2}, \ldots<\lambda_{N s+N t}\right)$. Figure C.1 represents the various situations 
occuring as the straight line goes across the body. Each point in this figure represents an intersection between the line and the body. A connection between two points indicates the crossing of the body by the straight line; the lack of connection corresponds to the crossing of the outer of the body by the straight line. The symbols $t, S 1, S 2$ correspond to the torus, the first sphere encountered and the second sphere encountered respectively. The symbols $\mathrm{C} 1$ and $\mathrm{C} 2$ correspond to the conditions or inequalities:

$\mathrm{C} 1:\left|x_{1}+\lambda_{2}\left(x_{2}-x_{1}\right)\right|>R_{2} /\left(1+R_{2}\right)$

$\mathrm{C} 2:\left(y_{1}-x_{1}\left(y_{2}-y_{1}\right) /\left(x_{2}-x_{1}\right)\right)^{2}+\left(z_{1}-x_{1}\left(z_{2}-z_{1}\right) /\left(x_{2}-x_{1}\right)\right)^{2}<\left(R_{3}-R_{2}\right)^{2}$

If all the points are connected, the chord length obeys:

$l=\left|\lambda_{N s+N t}-\lambda_{1}\right| \sqrt{A}$

If an interruption in the point sequence occurs between the points $i$ and $i+1$, the chord length obeys:

$l=\left(\left|\lambda_{i}-\lambda_{1}\right|+\left|\lambda_{N s+N t}-\lambda_{i+1}\right|\right) \sqrt{A}$ 


\section{Captions for figures}

Figure 1: Sphere (a), Dumbbell (b) and the Diabolo (c)

Figure 2a: CLD $D(l)$ of dumbbells for various $\delta$-values: $\delta=2$ dash-dot line (black); $\delta=1.6$ dotted line (red); $\delta=1$ dashed line (blue); $\delta=0.4$ solid line (green)

Figure 2b: CLD $D_{l}(l)$ of dumbbells for various $\delta$-values: $\delta=2$ dash-dot line (black); $\delta=1.6$ dotted line (red); $\delta=1$ dashed line (blue); $\delta=0.4$ solid line (green)

Figure 3a: CLD $D(l)$ of Diabolos for various $\mathrm{R}_{2}$-values: $\mathrm{R}_{2}=0$ dash-dot line (black); $\mathrm{R}_{2}=1$ dotted line (red); $\mathrm{R}_{2}=4$ dashed line (blue); $\mathrm{R}_{2}=100$ solid line (green)

Figure 3b: CLD $D_{l}(l)$ of Diabolos for various $\mathrm{R}_{2}$-values: $\mathrm{R}_{2}=0$ dash-dot line (black); $\mathrm{R}_{2}=1$ dotted line (red); $\mathrm{R}_{2}=4$ dashed line (blue); $\mathrm{R}_{2}=100$ solid line (green)

Figure 4a: $l_{t h} / l_{M C}$ against $\delta$ for dumbbells. Red circle: $l_{t h}=4 V / S^{*}$; green plus: $l_{t h}=4 V / S$; Blue star: $l_{t h}=4\left(V+\left(V^{*}-V\right) / 3\right) / S^{*}$; black plus: $l_{t h}=V /\left\langle S_{P}\right\rangle$.

Figure 4b: $l_{t h} / l_{M C}$ against $R_{2}$ for diabolos. Red circle: $l_{t h}=4 V / S^{*}$; green plus: $l_{t h}=4 V / S$; Blue star: $l_{t h}=4\left(V+\left(V^{*}-V\right) / 3\right) / S^{*}$; black plus: $l_{t h}=V /\left\langle S_{P}\right\rangle$.

Figure 5: $\left\langle|R|^{2}\right\rangle$ as a function of $q R_{l}$ for a diabolo. Dotted line: calculated from distance distribution (MCS); line: from CLD (MCS). red: $R_{2}=100$; blue: $R_{2}=1$; black: $R_{2}=0.02$. $\lambda=450 \mathrm{~nm} . R_{l}=0.2 \mu \mathrm{m}$.

Figure A.1: intersection between a straight line and a sphere

Figure B.1: intersection between a straight line and a dumbbell (view 1)

Figure B.2: intersection between a straight line and a dumbbell (view 2)

Figure C.1: intersection points and chords: straight line crossing the Diabolo. See the text for the explanation. 


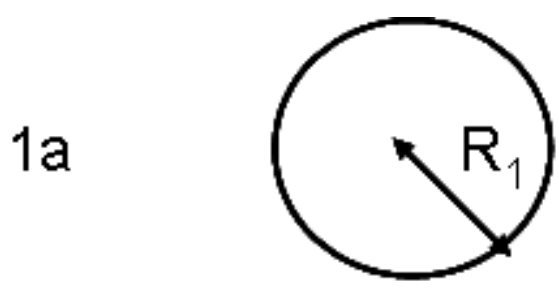

$1 b$
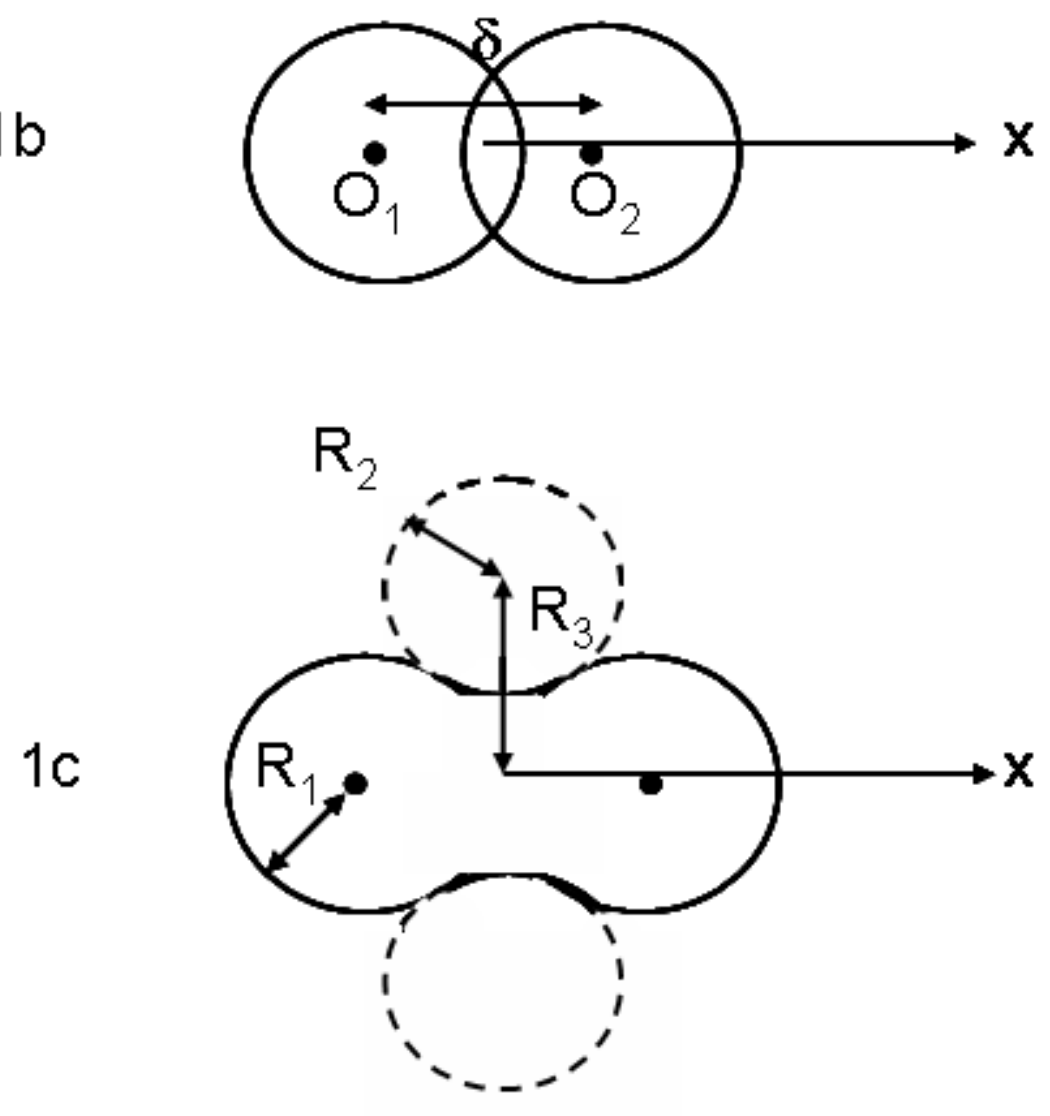

Figure 1: Sphere (a), Dumbbell (b) and the Diabolo (c) 


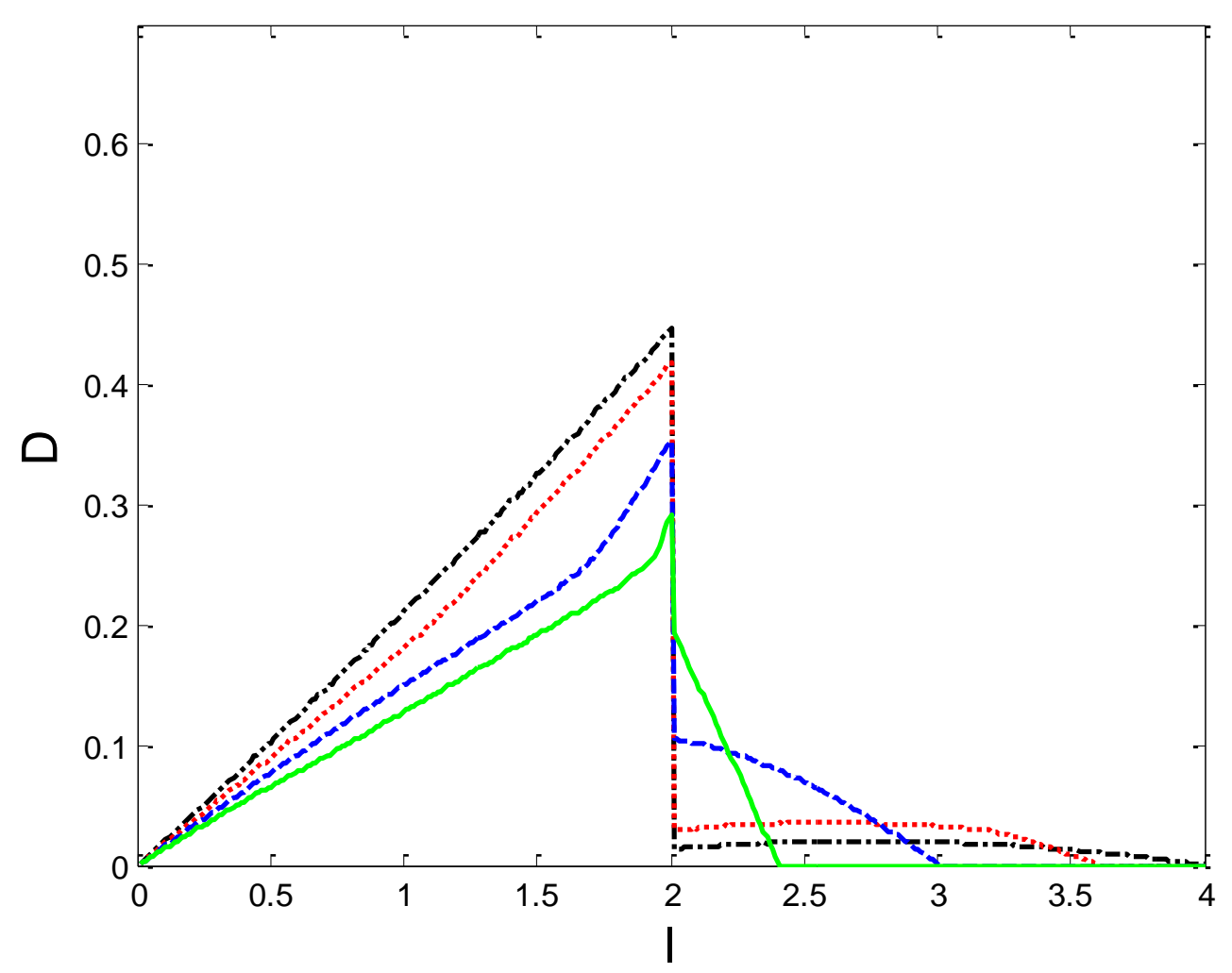

Figure 2a: CLD $D(l)$ of dumbbells for various $\delta$-values: $\delta=2$ dash-dot line (black); $\delta=1.6$ dotted line (red); $\delta=1$ dashed line (blue); $\delta=0.4$ solid line (green) 


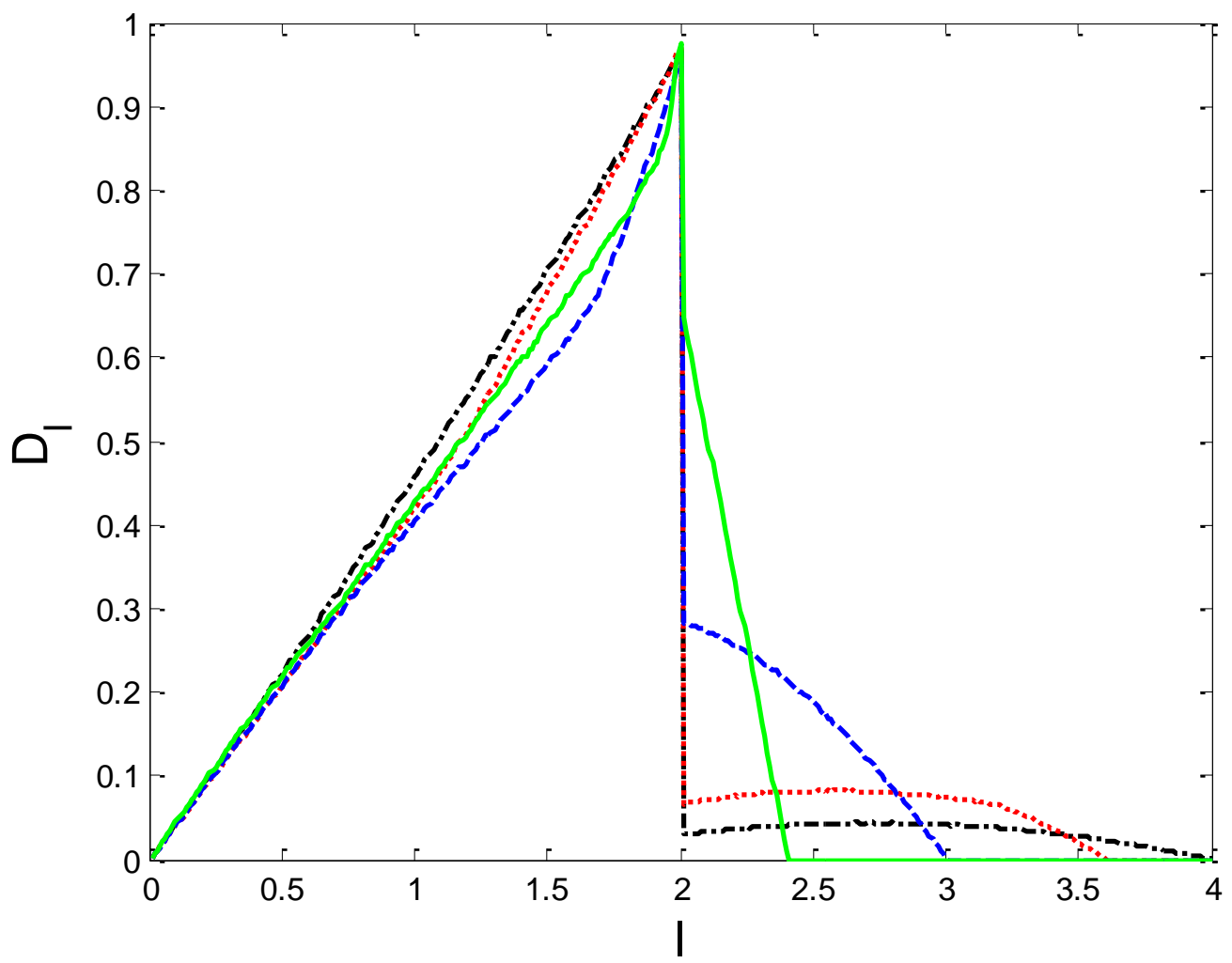

Figure 2b: CLD $D_{l}(l)$ of dumbbells for various $\delta$-values: $\delta=2$ dash-dot line (black); $\delta=1.6$ dotted line (red); $\delta=1$ dashed line (blue); $\delta=0.4$ solid line (green) 


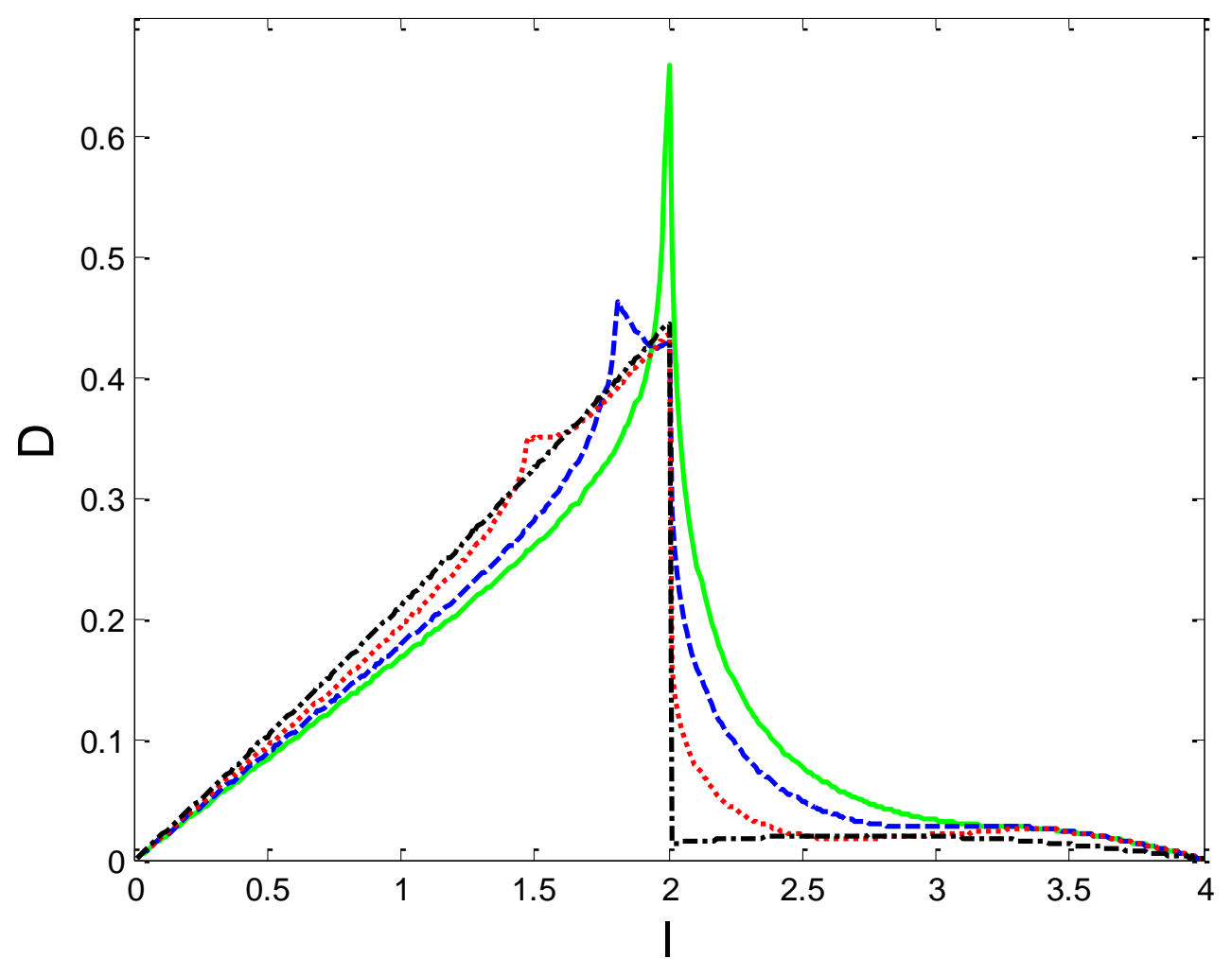

Figure 3a: CLD $D(l)$ of Diabolos for various $\mathrm{R}_{2}$-values: $\mathrm{R}_{2}=0$ dash-dot line (black); $\mathrm{R}_{2}=1$ dotted line (red); $\mathrm{R}_{2}=4$ dashed line (blue); $\mathrm{R}_{2}=100$ solid line (green) 


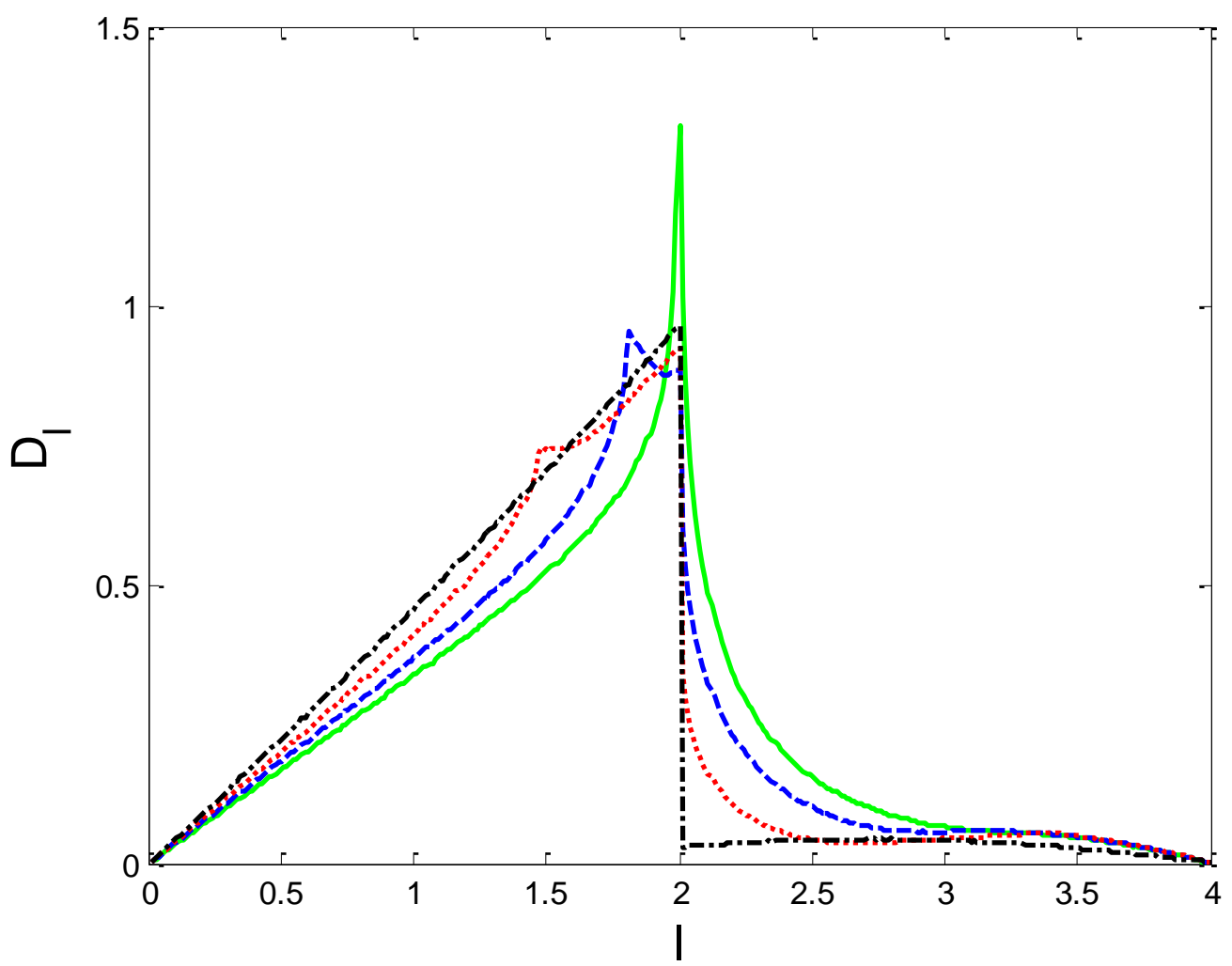

Figure 3b: CLD $D_{l}(l)$ of Diabolos for various $\mathrm{R}_{2}$-values: $\mathrm{R}_{2}=0$ dash-dot line (black); $\mathrm{R}_{2}=1$ dotted line (red); $\mathrm{R}_{2}=4$ dashed line (blue); $\mathrm{R}_{2}=100$ solid line (green) 


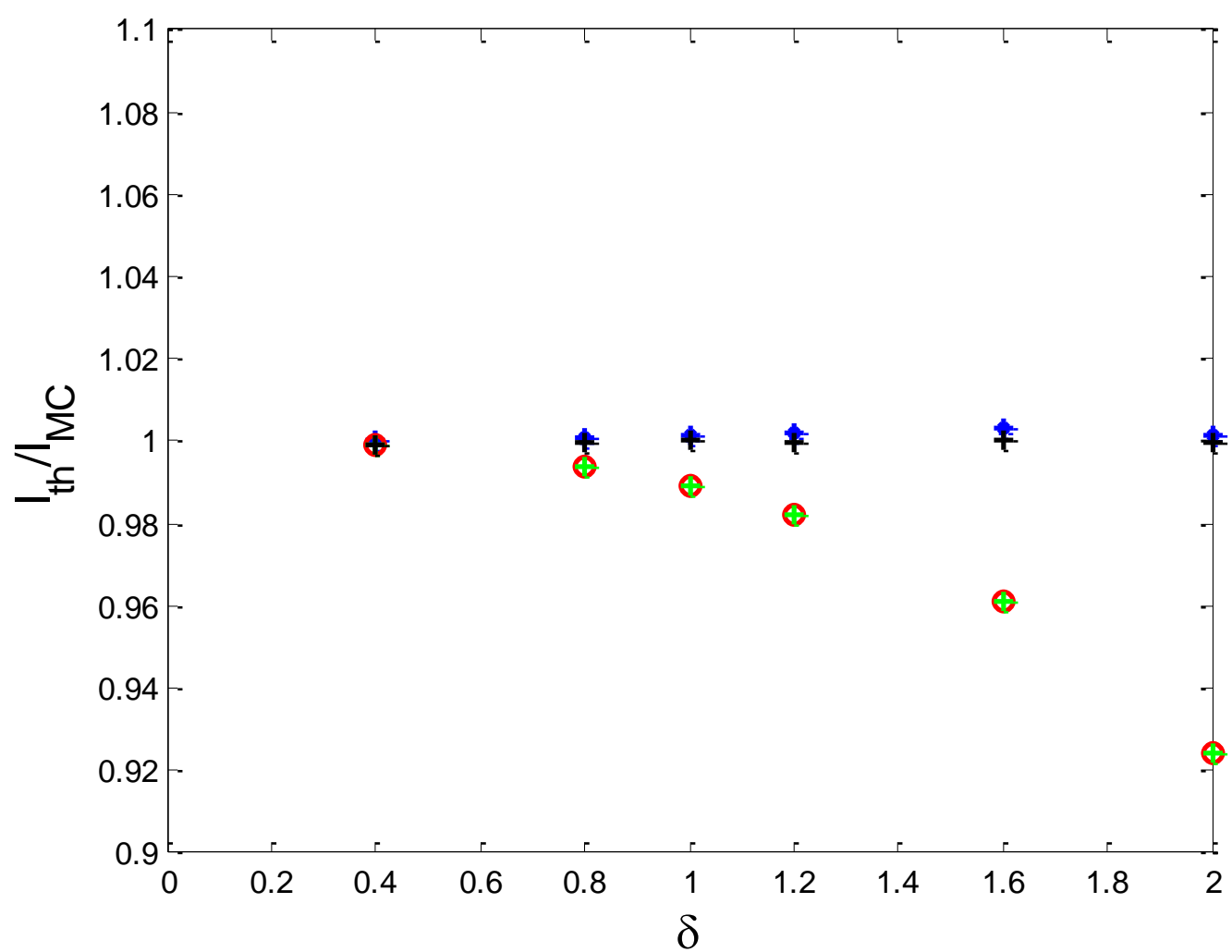

Figure 4a: $l_{t h} / l_{M C}$ against $\delta$ for dumbbells. Red circle: $l_{t h}=4 V / S^{*}$; green plus: $l_{t h}=4 V / S$; Blue star: $l_{t h}=4\left(V+\left(V^{*}-V\right) / 3\right) / S^{*}$; black plus: $l_{t h}=V /\left\langle S_{P}\right\rangle$. 


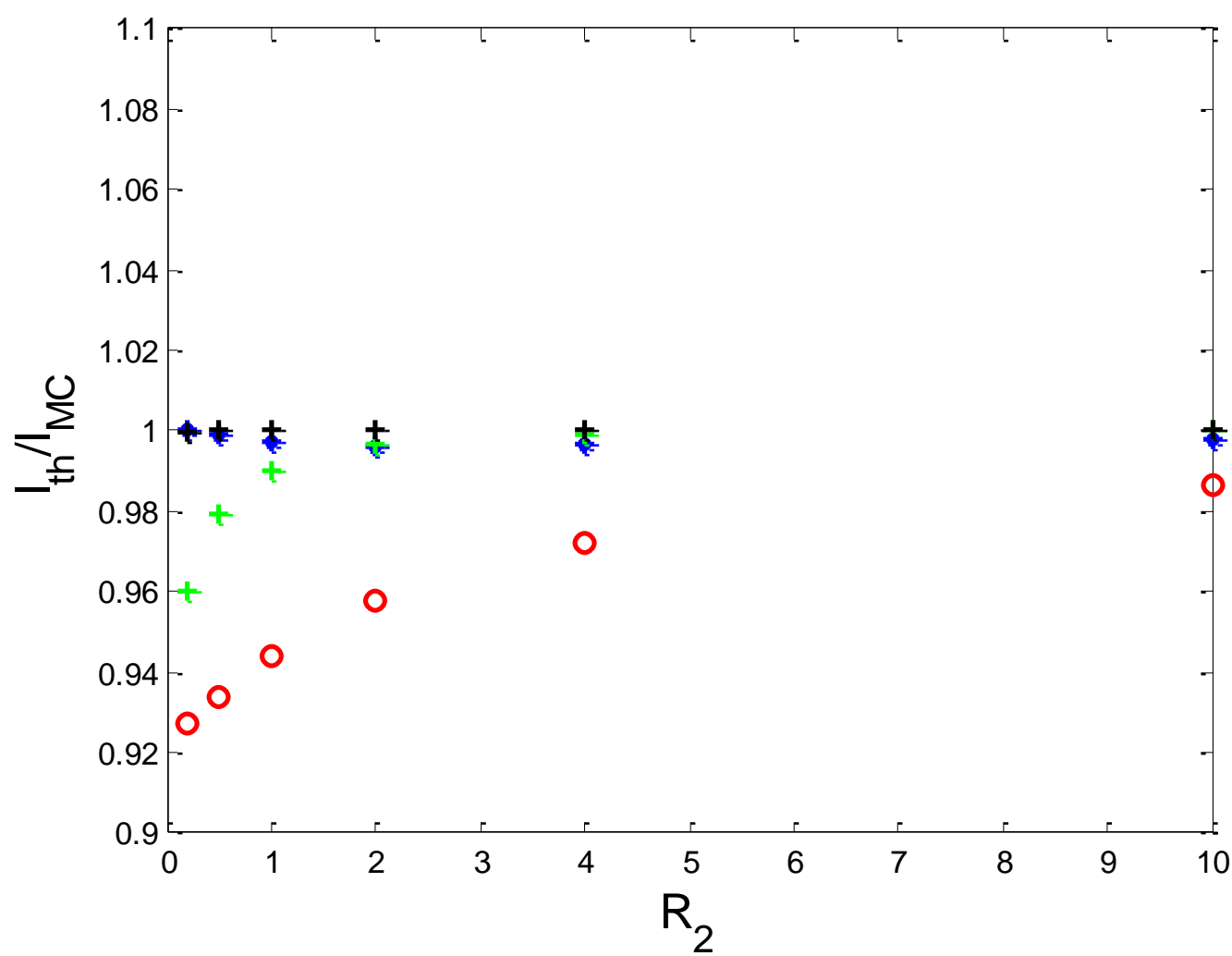

Figure 4b: $l_{t h} / l_{M C}$ against $R_{2}$ for diabolos. Red circle: $l_{t h}=4 V / S^{*}$; green plus: $l_{t h}=4 V / S$; Blue star: $l_{t h}=4\left(V+\left(V^{*}-V\right) / 3\right) / S^{*}$; black plus: $l_{t h}=V /\left\langle S_{P}\right\rangle$. 


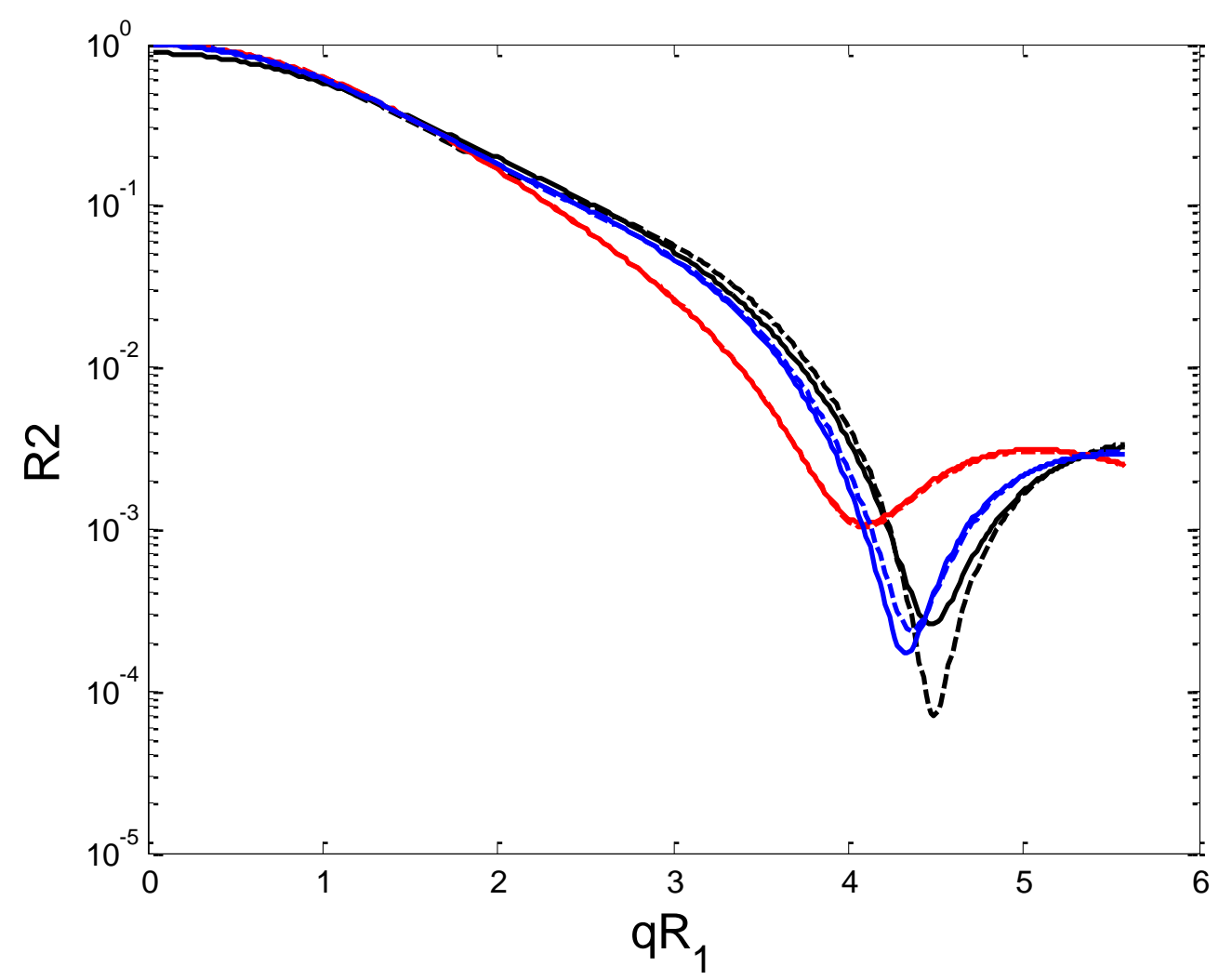

Figure 5: $\left\langle|R|^{2}\right\rangle$ as a function of $q R_{1}$ for a diabolo. Dotted line: calculated from distance distribution (MCS); line: from CLD (MCS). red: $R_{2}=100$; blue: $R_{2}=1$; black: $R_{2}=0.02$. $\lambda=450 \mathrm{~nm} . R_{l}=0.2 \mu \mathrm{m}$. 


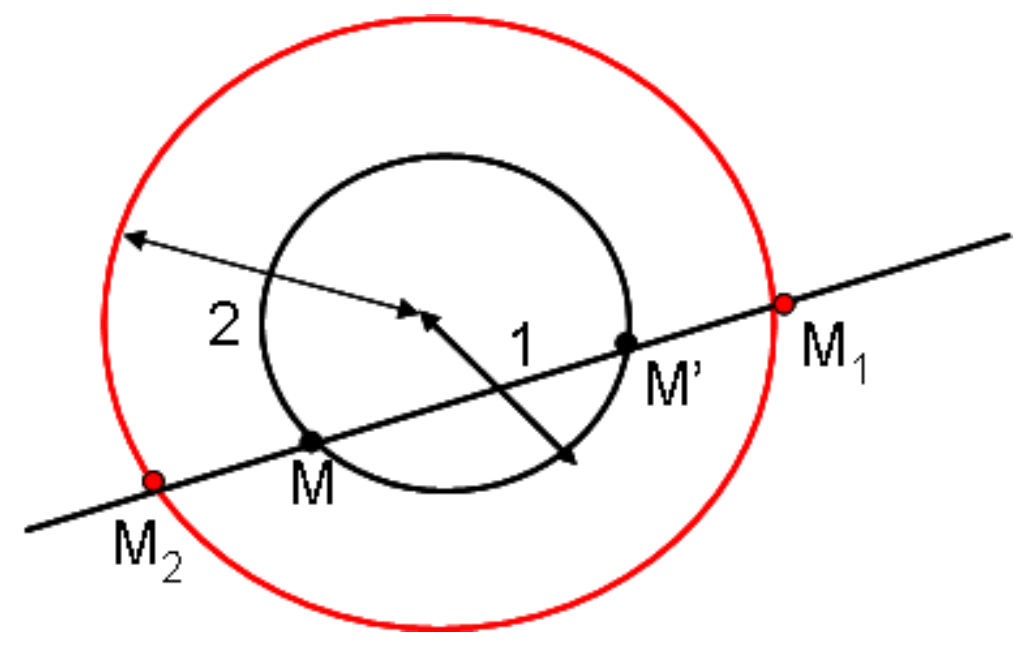

Figure A.1: intersection between a straight line and a sphere 


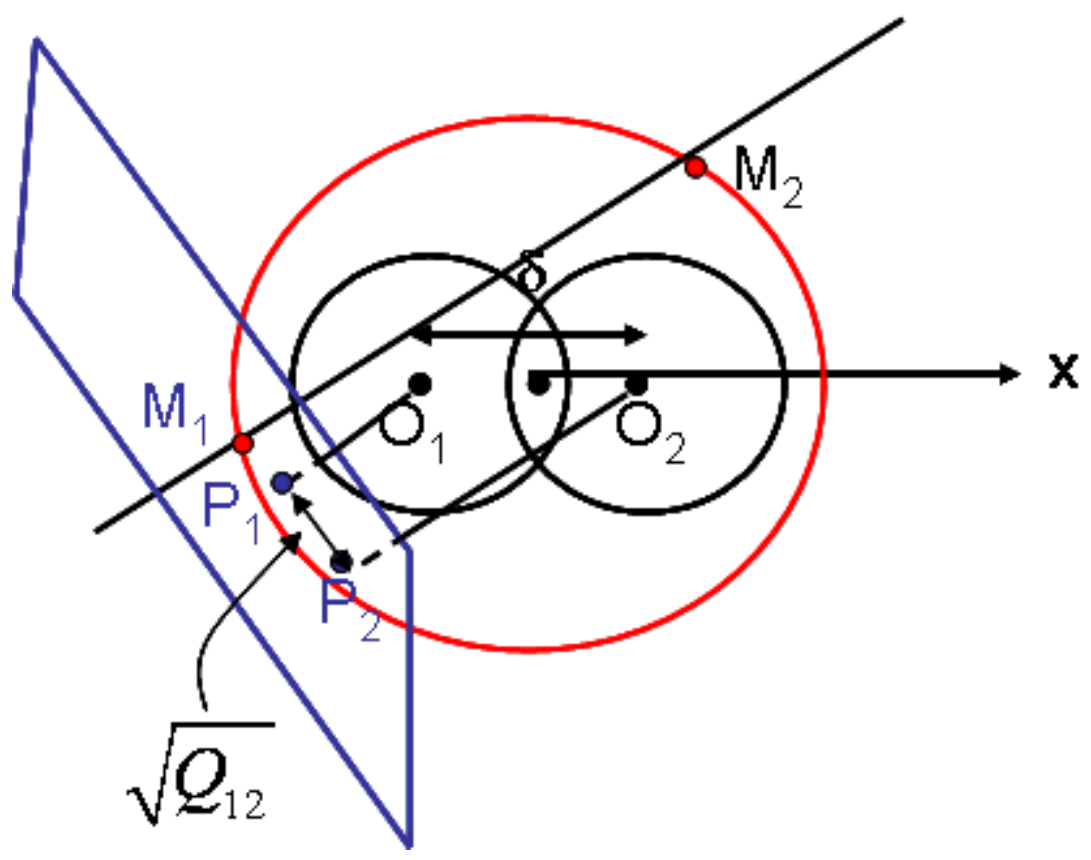

Figure B.1: intersection between a straight line and a dumbbell (view 1)

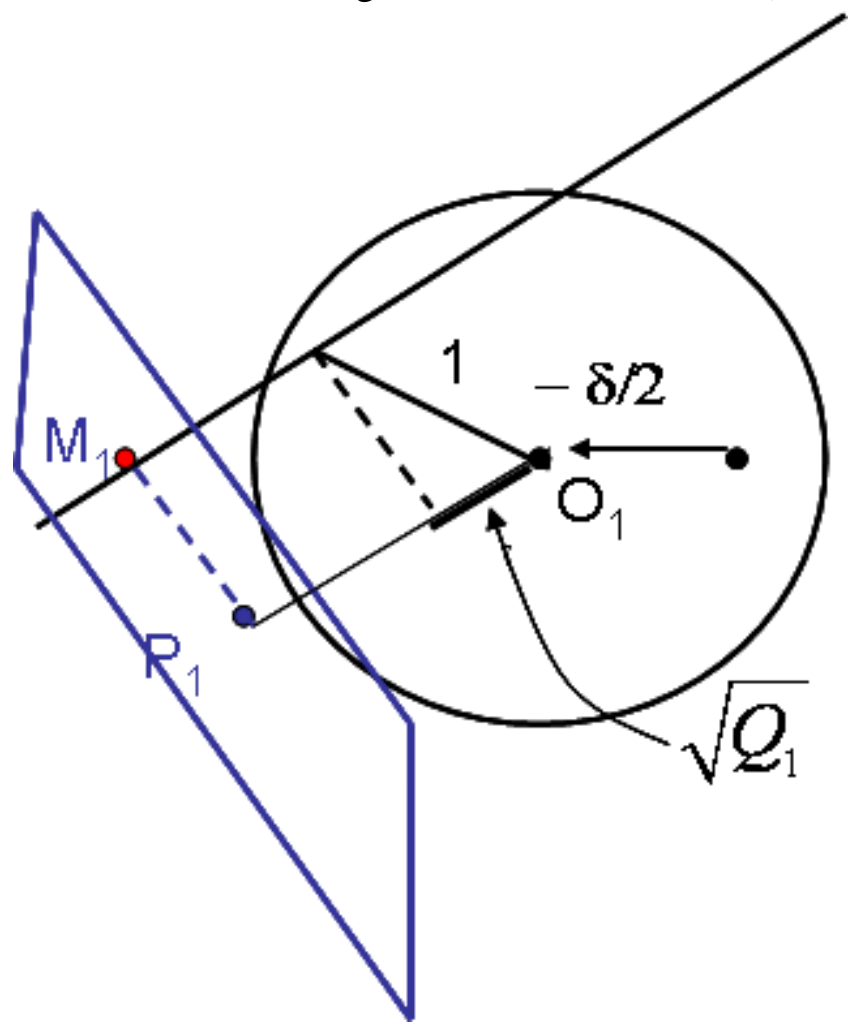

Figure B.2: intersection between a straight line and a dumbbell (view 2) 


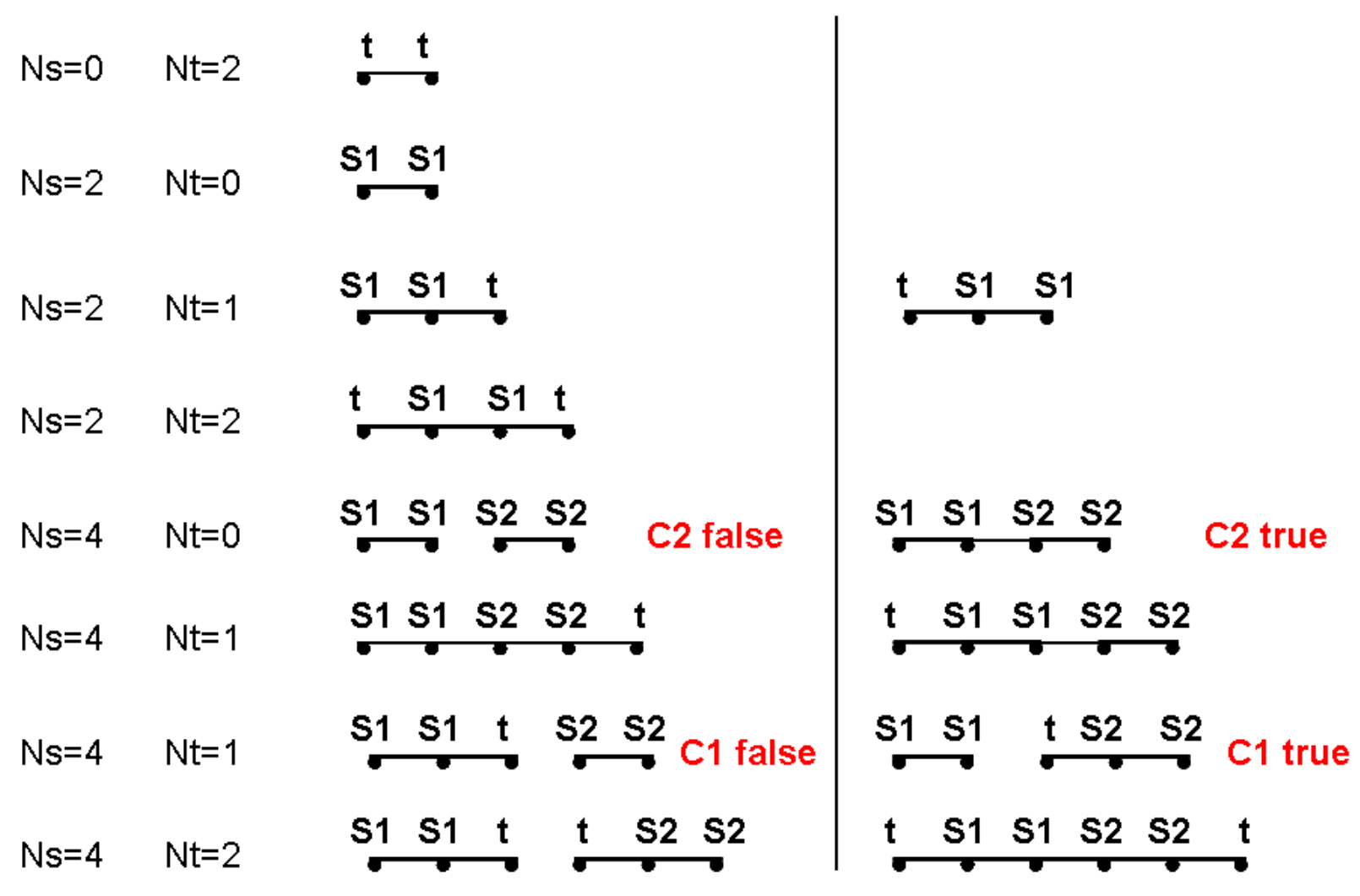

Figure C.1: intersection points and chords: straight line crossing the Diabolo. See the text for the explanation. 


\section{Caption for table}

Table 1: ratio $N I / N T$ of chord numbers for various values of $\delta$ (dumbbells) and $R_{2}$ (diabolos) 


\begin{tabular}{|l|l|l|l|l|l|l|l|l|}
\hline $\boldsymbol{\delta}$ & 0.4 & 0.8 & 1 & 1.2 & 1.6 & 2 & & \\
\hline $\boldsymbol{N} / \boldsymbol{N} \boldsymbol{T}$ & 0.3000 & 0.3480 & 0.3709 & 0.3930 & 0.4324 & 0.4622 & & \\
\hline $\boldsymbol{R}_{2}$ & 0.02 & 0.2 & 0.5 & 1 & 2 & 4 & 10 & 100 \\
\hline $\boldsymbol{N} / \boldsymbol{N T}$ & 0.4623 & 0.4636 & 0.4669 & 0.4720 & 0.4789 & 0.4859 & 0.4931 & 0.4992 \\
\hline
\end{tabular}

Table 1: ratio $N / / N T$ of chord numbers for various values of $\delta$ (dumbbells) and $R_{2}$ (diabolos) 